\title{
Ability to cause erythema migrans differs between Borrelia burgdorferi sensu lato isolates
}

\author{
Ellen Tijsse-Klasen ${ }^{1 *}$, Nenad Pandak ${ }^{2 *}$, Paul Hengeveld ${ }^{1}$, Katsuhisa Takumi ${ }^{1}$, Marion PG Koopmans ${ }^{1,3}$ \\ and Hein Sprong ${ }^{1}$
}

\begin{abstract}
Background: Lyme borreliosis is a tick-borne disease caused by Borrelia burgdorferi sensu lato. The variety of characteristic and non-specific clinical manifestations is partially explained by its genetic diversity. We investigated the ability of $B$. burgdorferi $s$ isolates to cause erythema migrans.

Methods: The genetic constellation of isolates from ticks was compared to isolates found in erythema migrans. PCR and sequence analysis was performed on the plasmid-encoded ospC and the chromosomal 5S-23S rDNA spacer region (IGS).

Results: Seven different $B$. burgdorferi sl genospecies were identified in 152 borrelia isolates from ticks and erythema migrans biopsies. B afzelii (51\%) and B. garinii (27\%) were the most common in ticks. From the 44 sequences obtained from erythema migrans samples 42 were B. afzelii, one B. garinii and one B. bavariensis. Significant associations with erythema migrans formation were found for four IGS and two ospC types. Five from 45 osp C types were associated with more than one genospecies.

Conclusions: $B$. burgdorferi sl isolates differ in their propensity to cause erythema migrans. These differences were also found within genospecies. In other words, although B. afzelii was mostly associated with erythema migrans, some $B$. afzelii isolates had a low ability to cause erythema migrans. Our data further support the occurrence of plasmid exchange between borrelia genospecies under natural conditions.
\end{abstract}

Keywords: Lyme borreliosis, Erythema migrans, Molecular epidemiology, Virulence marker

\section{Background}

Lyme borreliosis is the most common tick-borne infectious disease in North America and in countries with moderate climates in Eurasia. Lyme borreliosis is caused by several members of the Borrelia burgdorferi sensu lato (sl) complex. Based on the genetic diversity, this large family of spirochetes has been subdivided into 19 different taxonomic entities or genospecies, of which several have been shown to cause disease in humans including B. burgdorferi sensu stricto (ss), B. afzelli and B. garinii [1]. Lyme borreliosis can present with a variety of typical clinical manifestations. An early sign of Lyme borreliosis is erythema migrans (EM), which

\footnotetext{
* Correspondence: Ellen.TijsseKlasen@gmail.com; NPandak@gmail.com ${ }^{1}$ Centre for Infectious Disease Control, National Institute for Public Health and the Environment (RIVM), P.O. Box 1, Bilthoven 3720BA, Netherlands 'Department of Infectious Diseases, General Hospital "Dr. Josip Bencevic", Slavonski Brod, Croatia

Full list of author information is available at the end of the article
}

presents as a circular expanding skin rash. In a later stage, infections can present with arthritis, carditis or acrodermatitis chronica atrophicans (ACA), as well as neurological symptoms, non-specific symptoms such as fatigue, generalized pain and cognitive complaints [2]. All late stages of Lyme borreliosis can occur without preceding EM.

It has been proposed that the variability in the clinical manifestations of Lyme borreliosis can be partly explained by the involvement of different $B$. burgdorferi sl genospecies $[1,3,4]$. Lyme arthritis and carditis have mainly been associated with $B$. burgdorferi ss, whereas $B$. garinii has been associated preferentially with neuroborreliosis [5]. Erythema migrans can be caused by several $B$. burgdorferi sl genospecies but $B$. afzelii seems to be overrepresented in this group [6]. B. afzelii is also the genospecies most commonly associated with ACA, although B. garinii and B. burgdorferi ss have also been isolated from ACA cases

\section{Biomed Central}


[6,7]. Even within genospecies, differences in pathogenicity between $B$. burgdorferi ss strains have been shown [8-10]. However, despite numerous attempts, the various manifestations of Lyme borreliosis in humans cannot conclusively be attributed to infections with one specific genospecies of $B$. burgdorferi sl [11-14]. Understanding the possible differential pathogenicity between and within different borrelia genospecies is important because it will improve understanding of disease etiology and facilitate laboratory diagnostics.

Here, we investigated whether different genospecies and different members within a genospecies differ in their propensity to cause a typical manifestation of Lyme borreliosis, namely EM. The relative abundance of different $B$. burgdorferi sl genotypes in EM skin samples does not only depend on their ability to cause $\mathrm{EM}$, but also on their relative abundance in the tick population. Therefore, the genetic divergence of genospecies found in EM skin samples was compared to the genetic divergence of Borrelia in local tick populations. We investigated which typing methods could predict EM formation best: genospecies determination, IGS- or $\operatorname{ospC}$ haplotyping.

The rrfA-rrlB (5S-23S) rDNA intergenic spacer (IGS) has been used extensively to identify B. burgdorferi sl genospecies around the world and provides a good resolution within genospecies [15-20]. While IGS is a suitable marker to differentiate genospecies, unlike other, mainly surface exposed genes, it is not a virulence gene [21]. Outer surface protein $\mathrm{C}(\operatorname{ssp} C)$, which is encoded on the cp26 plasmid, is the best studied of these [22,23]. Although its exact function is still unknown, it has been shown to play an important role in early infection [22,24]. Several studies from the United States found that only a selection of $\operatorname{ssp} C$ types found in ticks are also detected in patient material, especially in disseminated infections [8-10]. More recent studies, however, have extended the number of pathogenic ospC genotypes substantially $[25,26]$. The involvement of $o s p C$ in early Lyme borreliosis makes it an interesting marker for studying the Borrelia genotypes involved in EM and $\operatorname{sp} p$ types might be more strongly correlated with EM than IGS types.

\section{Methods}

\section{Collection of skin biopsies and serum samples}

During the period 2008-2011, patients presenting in one of the three participating hospitals in Slavonski Brod, Rijeka or Čakovec with erythema migrans (EM) were enrolled in the study. EM was diagnosed by experienced physicians and each patient underwent skin biopsy of the EM active edge. The area of the biopsy was infiltrated with $1.5 \mathrm{ml}$ of $1 \%$ lidocain hydrochloride solution after which the incisional skin biopsy was performed. Skin samples were stored in $70 \%$ ethanol at $-20^{\circ} \mathrm{C}$ until further analysis. The study was approved by the General Hospital Slavonski Brod Ethic Committee and all patients signed an informed consent form.

\section{Collection of ticks}

During the period March-June 2011, ticks were collected at three different sites located 15 to $20 \mathrm{~km}$ east, north and west of Slavonski Brod city limits (Figure 1). Ticks were collected by blanket dragging, placed in individual tubes and stored at $-20^{\circ} \mathrm{C}$ until analysis.

\section{DNA extraction}

DNA of skin biopsies was extracted using the DNeasy Blood and Tissue Kit (Qiagen, Hilden, Germany) protocol for animal tissue according to the manufacturer's instructions.

DNA from vegetation ticks was extracted by alkaline lysis as described earlier [27]. If PCR inhibition was observed in conventional PCR, samples were further purified. Supernatant or the original tick was used to extract DNA using a DNeasy Blood and Tissue kit (Qiagen, Hilden, Germany) according to the manufacturer's protocols for body fluids or engorged ticks, respectively.

\section{Detection and identification of $B$. burgdorferi sl}

B. burgdorferi sl was detected using a duplex qPCR based on the OspA and flagellin genes and carried out on a LightCycler 480 instrument (Roche Diagnostics Nederland B.V, Almere, the Netherlands) [28]. Reactions were done in a final volume of $20 \mu \mathrm{l}$ with iQ multiplex Powermix, $3 \mu \mathrm{l}$ of template DNA and $0.2 \mu \mathrm{M}$ for all primers and probes (Table 1) except for OspA_probe, which was used at a final concentration of $0.1 \mu \mathrm{M}$. After iTaq DNA polymerase activation at $95^{\circ} \mathrm{C}$ for 5 minutes, 60 cycles of

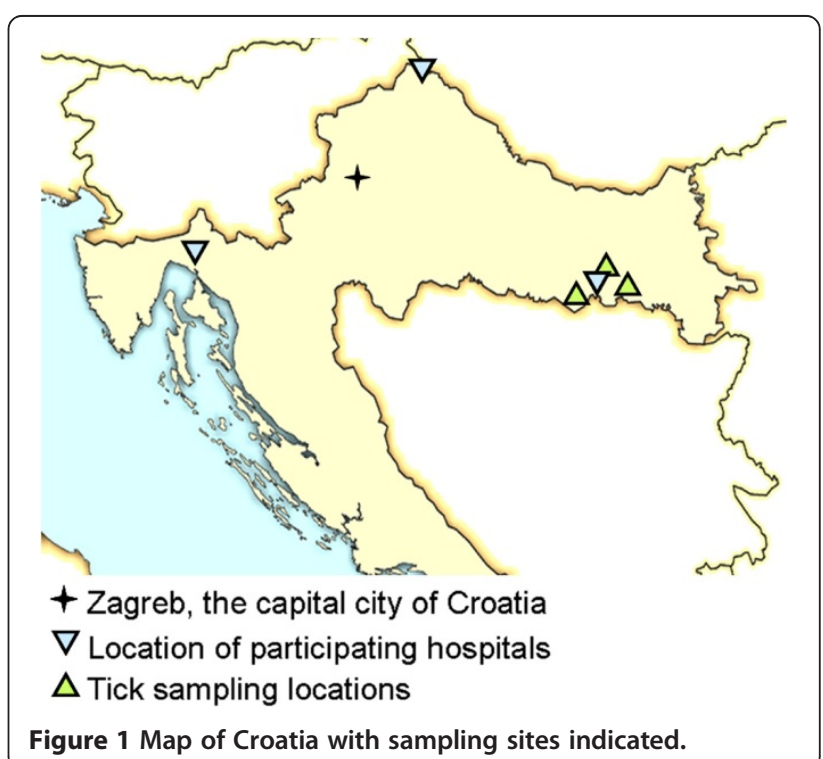


Table 1 DNA sequences of primer and probes

\begin{tabular}{ll}
\hline Oligo name & Sequence $\mathbf{( 5}^{\prime}$-3') \\
\hline Duplex qPCR & \\
OspA_F & AATATTATTGGGAATAGGTCTAA \\
OspA_R & CTITGTCTIITCTTTRCTTACAAG \\
FlaB-F & CAGAIAGAGGTTCTATACAIATTGAIATAGA \\
FlaB-Rc/t & GTGCATTTGGTAIATTGYGC \\
OspA_probe & FAM-AAGCAAAATGTTAGCAGCCTTGA-BHQ-1 ${ }^{\text {TM }}$ \\
FlaB-probe & JOE-CAACTIACAGAIGAAAXTAAIAGAATTGCTGCTGACA \\
IGS PCR & \\
5S & GAGTTCGCGGGAGAGTAGGTTATTGCC \\
23S & TCAGGGTACTTAGATGGTTCACTTCC \\
OspC PCR & \\
OC6 & AAAGAATACATTAAGTGCGATATT \\
OC602 & GGGCTTGTAAGCTCTTAACTG
\end{tabular}

FAM: 6-carboxyfluorescein; JOE: -carboxy-4',5'-dichloro-2', 7'-dimethoxyfluorescein.

$95^{\circ} \mathrm{C}$ for 5 seconds, $60^{\circ} \mathrm{C}$ for 35 seconds (ramp rate $2,2^{\circ} \mathrm{C} / \mathrm{s}$ and a single point measurement at $\left.60^{\circ} \mathrm{C}\right) 37^{\circ} \mathrm{C}$ for 20 seconds were run. Quantification cycle $(\mathrm{Cq})$ values were calculated with the LightCycler 480 software (release 1.5.0. SP3). Positive controls (B. burgdorferi ss B31) and negative water controls were used on every plate tested. Samples positive for one or both targets were scored positive for B. burgdorferi sl.

qPCR positive samples were subjected to conventional PCR on the 5S-23S rRNA (rrfA-rrlB) intergenic spacer region (IGS) and ospC gene. IGS PCR was done using primers $5 \mathrm{~S}$ and $23 \mathrm{~S}$ (Table 1) at final concentrations of $0.5 \mu \mathrm{M}$. The PCR was performed in a total reaction volume of $25 \mu \mathrm{l}$ using HotStarTaq master mix (Qiagen, Hilden, Germany) under following conditions: $15 \mathrm{~min}$ $94^{\circ} \mathrm{C}$, then cycles of $20 \mathrm{~s} 94^{\circ} \mathrm{C}, 30 \mathrm{~s} 70^{\circ} \mathrm{C}, 30 \mathrm{~s} 72^{\circ} \mathrm{C}$ lowering the annealing temperature $1^{\circ} \mathrm{C}$ each cycle until reaching $60^{\circ} \mathrm{C}$, then 40 cycles at this annealing temperature and a final elongation step for $7 \mathrm{~min}$ at $72^{\circ} \mathrm{C}$.

Conventional PCR on the ospC gene was performed using primers OC6 and OC602 at final concentrations of 0.4 $\mu \mathrm{M}$ [29]. The PCR was performed using HotStarTaq master mix under following conditions: $15 \mathrm{~min} 94^{\circ} \mathrm{C}$, then cycles of $30 \mathrm{~s} 94^{\circ} \mathrm{C}, 30 \mathrm{~s} 60^{\circ} \mathrm{C}, 50 \mathrm{~s} 72^{\circ} \mathrm{C}$ lowering the annealing temperature $1^{\circ} \mathrm{C}$ each cycle until reaching $50^{\circ} \mathrm{C}$, then 35 cycles at an annealing temperature of $53^{\circ} \mathrm{C}$ and a final elongation step for $7 \mathrm{~min}$ at $72^{\circ} \mathrm{C}$.

Amplicons from conventional PCRs were sequenced on both strands on a 3730 DNA Analyzer using ABI PRISM Big-Dye Terminator Cycle sequencing Ready Reaction kit (Applied Biosystems, Carlsbad, CA, USA).

B. burgdorferi sl genospecies were inferred from the IGS sequence by comparing the sequences with a large local database ( \pm 3000 entries) including, amongst others, all
IGS entries from GenBank and 170 IGS sequences from different samples confirmed by multi-locus sequence typing.

In order to minimize contamination, PCR proceedings were performed in three separate rooms, of which the DNA extraction room was kept at negative pressure and the reagent setup and sample addition rooms were kept at positive pressure. All rooms had airlocks.

\section{Genetic analysis}

Sequence quality was visually checked and sequences of both strands were assembled using Bionumerics software. IGS sequences were aligned together with a $B$. afzelii reference sequence from Genbank (strain PKo, Genbank entry CP002933). An IGS or ospC type was defined as a sequence diverging at least one nucleotide or gap from any other sequence in the dataset. The number of IGS types was determined based on the sequence fragment between position 438843 and 439146 of whole genome sequence of this reference strain. Likewise, osp $C$ sequences were aligned with a $B$. afzelii PKo reference strain (Genbank entry CP002934) between position 17065 and 17463 and $\operatorname{sp} C$ types were identified. Short or poor quality $o s p C$ sequences, of which some might have been caused by double infections or multiple plasmids, were rejected from further analysis. IGS and $o s p C$ types were numbered independent from their phylogenetic relationships.

\section{Statistical analysis}

The diversity of IGS and $o s p C$ types found in ticks was compared with that in EM skin samples using a binomial test of statistical significance. The hypothesis that certain IGS or ospC types are found equally often in EM skin samples and in tick samples was compared with the alternative that a certain IGS or ospC type is predominantly associated with one of the sample types. A likelihood ratio test with a degree of freedom of one was performed on this model. A certain IGS or ospC type is associated with ticks or EM skin samples if the $p$-value of the likelihood ratio test was less than 0.05 .

\section{Results}

\section{Collected samples}

During a four-year period, 67 patients presenting in one of the three participating hospitals were enrolled in the study. Forty-one DNA isolates from EM biopsies were taken at a hospital in Slavonski Brod, 16 in Čakovec and 10 in Rijeka. In the same period, 1573 ticks were collected for molecular analysis from three locations near Slavonski Brod (Figure 1). Of these, 1433 (91\%) were identified as adult Ixodes ricinus, 117 (7.4\%) as Dermacentor marginatus and $24(1.5 \%)$ as Haemaphysalis concinna. 


\section{Detection and identification of $B$. burgdorferi sl}

Using the duplex B. burgdorferi sl qPCR, 254 of 1432 I. ricinus ticks (18\%) were found positive for B. burgdorferi sl. All D. marginatus $(\mathrm{n}=117)$ and $H$. concinna ticks ( $\mathrm{n}=24$ ) were negative. B. burgdorferi sl positive I. ricinus were subjected to conventional PCR on both IGS and ospC followed by sequencing. Due to the lower sensitivity of conventional PCR compared to qPCR, only 108 IGS and 83 osp $C$ sequences were obtained from qPCR positive ticks. From 67 skin samples 47 (70\%) were found positive in duplex qPCR. Conventional PCR and sequencing on these samples yielded 44 IGS and 37 ospC sequences, respectively.

Borrelia genospecies were identified based on IGS sequences. Fifty-five of 108 sequenced isolates from ticks and 42 of 47 isolates from EM samples were identified as B. afzelii. Likewise, B. garinii was detected in 29 I. ricinus and one EM skin sample. B. bavariensis was only found in a single EM skin sample from Rijeka while isolates belonging to $B$. burgdorferi ss, $B$. valaisiana, B. lusitianae and B. spielmanii were only found in ticks $(\mathrm{n}=5,8,10$ and 1 , respectively) (Table 2).

In the following, the term isolate is used to describe a DNA sample isolated from a human or tick. This implies that an isolate may contain more than one B. burgdorferi sl genospecies.

In total, IGS alignment revealed 31 different IGS types, with each type diverging at least one nucleotide or gap from other sequences in the sample (Additional file 1: Figure S1). B. afzelii isolates in this study could be subdivided in 16 IGS types. Seven, three and two different IGS types were found for B. garinii, B. burgdorferi ss and $B$. valaisiana, respectively, while the remaining genospecies (B. bavariensis, B. lusitaniae, B. spielmanii) comprised a single IGS type. Four IGS types were exclusively found in EM samples, 18 only in tick samples and 9 in both sample types (Figure 2).

Table 2 Number of isolates from tick and EM samples and IGS and OspC types distribution across genospecies

\begin{tabular}{lccccc}
\hline Borrelia species & \multicolumn{2}{c}{ Isolates from } & & \multicolumn{2}{c}{ Haplotypes of } \\
\cline { 2 - 3 } \cline { 5 - 6 } & Ticks (n) & EM (n) & & IGS (\#) & OspC (\#) \\
\hline B. afzelii & 55 & 42 & & 16 & 23 \\
B. garinii & 29 & 1 & & 7 & 11 \\
B. burgdorferi ss & 5 & 0 & & 3 & 2 \\
B. valaisiana & 8 & 0 & & 2 & 5 \\
B. bavariensis & 0 & 1 & & 1 & $* *$ \\
B. lusitaniae & 10 & 0 & & 1 & 5 \\
B. spielmanii & 1 & 0 & & 1 & 1 \\
Total & 108 & 44 & 31 & $47\left(45^{*}\right)$ \\
\hline
\end{tabular}

$\mathrm{n}$ : number of isolates; \#: number of haplotypes. * Some OspC haplotypes were found in several genospecies, therefore entries in this column do not add up to $45 . * *$ OspC could not be determined.
OspC alignment revealed 45 different ospC types (Additional file 2: Figure S2) of which four were found exclusively in EM skin samples, 34 only in tick samples and 7 in both sample types.

In 66 samples from ticks and 33 samples from EM both IGS and $\operatorname{ssp} C$ were sequenced. Analysis of these samples showed that five ospC types (numbers 5, 20, 33, 42 and 7; Figure 3) were found in two or more genospecies.

\section{Correlation of B. burgdorferi sI IGS and ospC types with EM}

Only 13 of 31 IGS types that were identified in this study were detected in skin biopsies from erythema migrans patients. The skin samples in this study were collected in three different locations throughout Croatia (Slavonski Brod, Rijeka and Čakovec) but ticks were only collected in proximity to Slavonski Brod. Considering all collected skin samples, IGS types 21, 18, 6 and 7 (order as in Figure 2A) were found significantly more often in patient samples than would have been expected on basis of the Borrelia types found in ticks. In contrary, IGS types 2, 14, 3 and 25 were significantly less often found in EM skin samples. When only samples from Slavonski Brod were used, two IGS types remained significantly associated with EM (Figure 2B) and two were found significantly less often in EM than expected.

The second genetic marker used in this study, ospC, is encoded on a plasmid of $B$. burgdorferi. Of the 44 different ospC types that were detected, 24 were found in B. afzelii but not necessarily restricted to this genospecies. There was a strong correlation of $o s p C$ type with EM. This correlation was significant for ospC types 5 and 3 (order as in Figure 2B) and types 39 and 20 were found significantly less often than expected. These associations stayed significant for $o s p C$ types 5 and 20 when only skin samples from Slavonski Brod were analysed (Figure 2D).

\section{Discussion}

Three tick species were collected during this study, all of which had been reported from the northern Balkan region before [30]. The majority of the ticks collected were I. ricinus which is the main vector for Lyme borreliosis in Europe. The infection rates of I. ricinus were similar to those reported in earlier studies in Croatia and other European countries [31-33] but much higher and lower infection rates were also reported from a neighbouring country $[34,35]$. Seven out of nine B. burgdorferi sl genospecies known to occur in Europe were identified and only B. bissettii and B. kurtenbachii were not found [1]. As in most European countries B. afzelii was the most common genospecies identified.

In EM skin biopsies three different B. burgdorferi sl genospecies were detected and the previously recognized overrepresentation of $B$. afzelii in the skin condition 


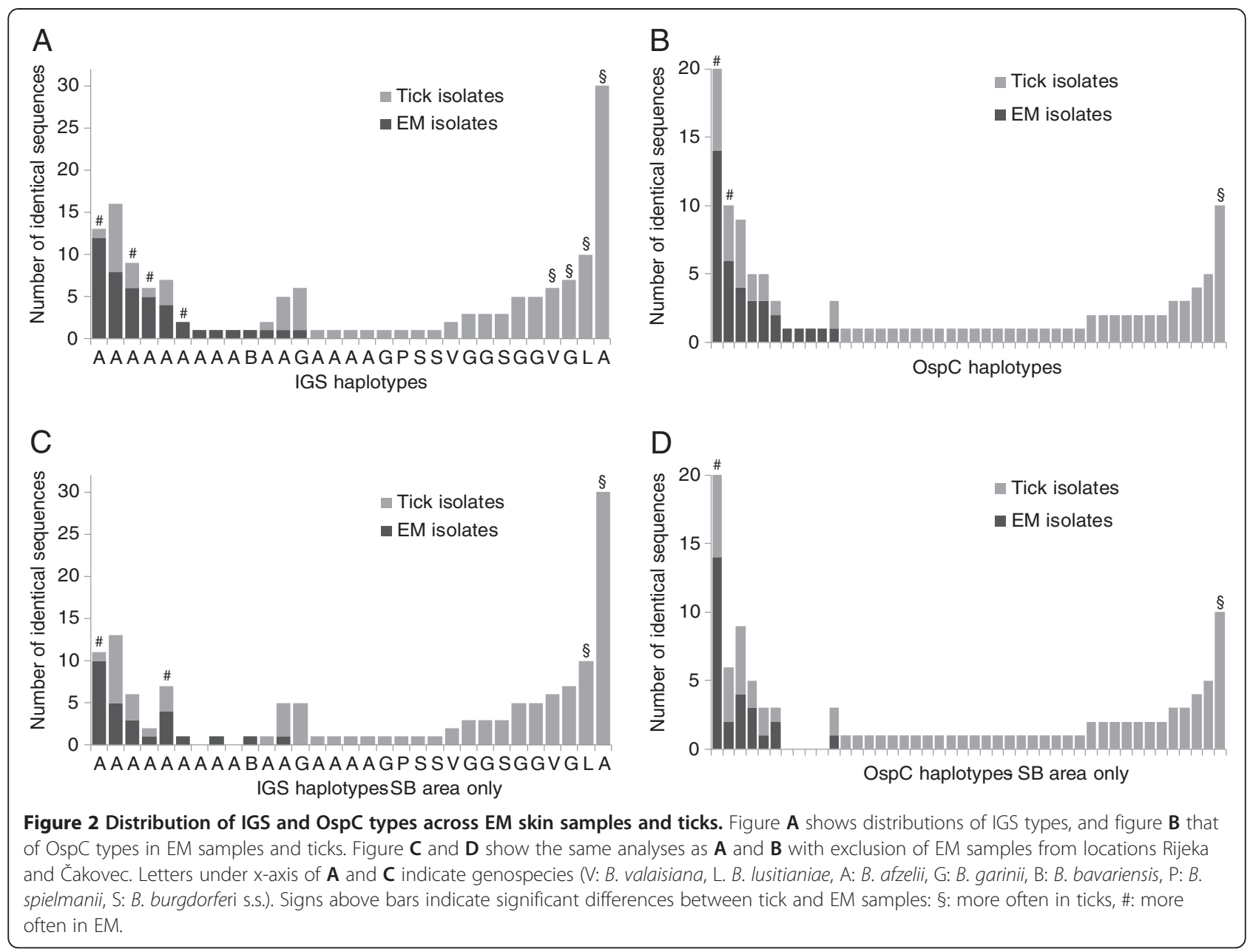

erythema migrans was confirmed $[4,6]$. More importantly, a strong correlation between certain IGS types within the B. afzelii cluster and EM were found.

In earlier studies, certain $o s p C$ types of $B$. burgdorferi ss have been shown to be associated with higher probabilities of invasiveness and human disease [8-10,36]. Lagal and co-workers found similar associations for B. afzelii and $B$. garinii strains in Europe but did not compare the frequency of the different $o s p C$ types in clinical samples to those in the tick population [37]. We expanded these observations to EM in Croatia, where a broad range of B. burgdorferi $\mathrm{sl}$ is present and supported our findings with substantial tick data. Correlations of EM with certain types of the plasmid-encoded marker osp $C$ were evident from our datasets. The expectation that this gene, due to its role in early infection, might show a stronger correlation to EM than IGS, could not be confirmed [24].

IGS type determination predicted the isolates propensity to cause EM better than genospecies determination alone. This is illustrated by the example that both the isolates with the highest and lowest propensity to cause EM belonged to B. afzelii genospecies. Significant positive and negative association with EM were more often found for IGS- than for $\operatorname{ssp} C$-typing (Figure 2) suggesting that IGS predicts EM formation better than ospC. This would imply that at least some of the genes responsible for EM formation are located on the chromosome or other non-exchangeable genomic elements.

As pointed out by Dykhuizen and co-workers it is important to use tick data alongside patient data to correctly estimate the propensity of strains to cause infection [36]. This was done in the present study by sampling ticks around the main study site, from which the majority of patient samples were obtained. As a geographic bias could not be excluded, analyses were done for the whole study population or the Slavonski Brod study population alone. When only Slavonski Brod samples were taken into account, fewer IGS and $o s p C$ types were significantly more or less often found in patients than expected than when the whole study population was used. However, even when only isolates from this site were analysed one ospC and two IGS types were significantly more often found in patients than expected. Four patients from Čakovec and 


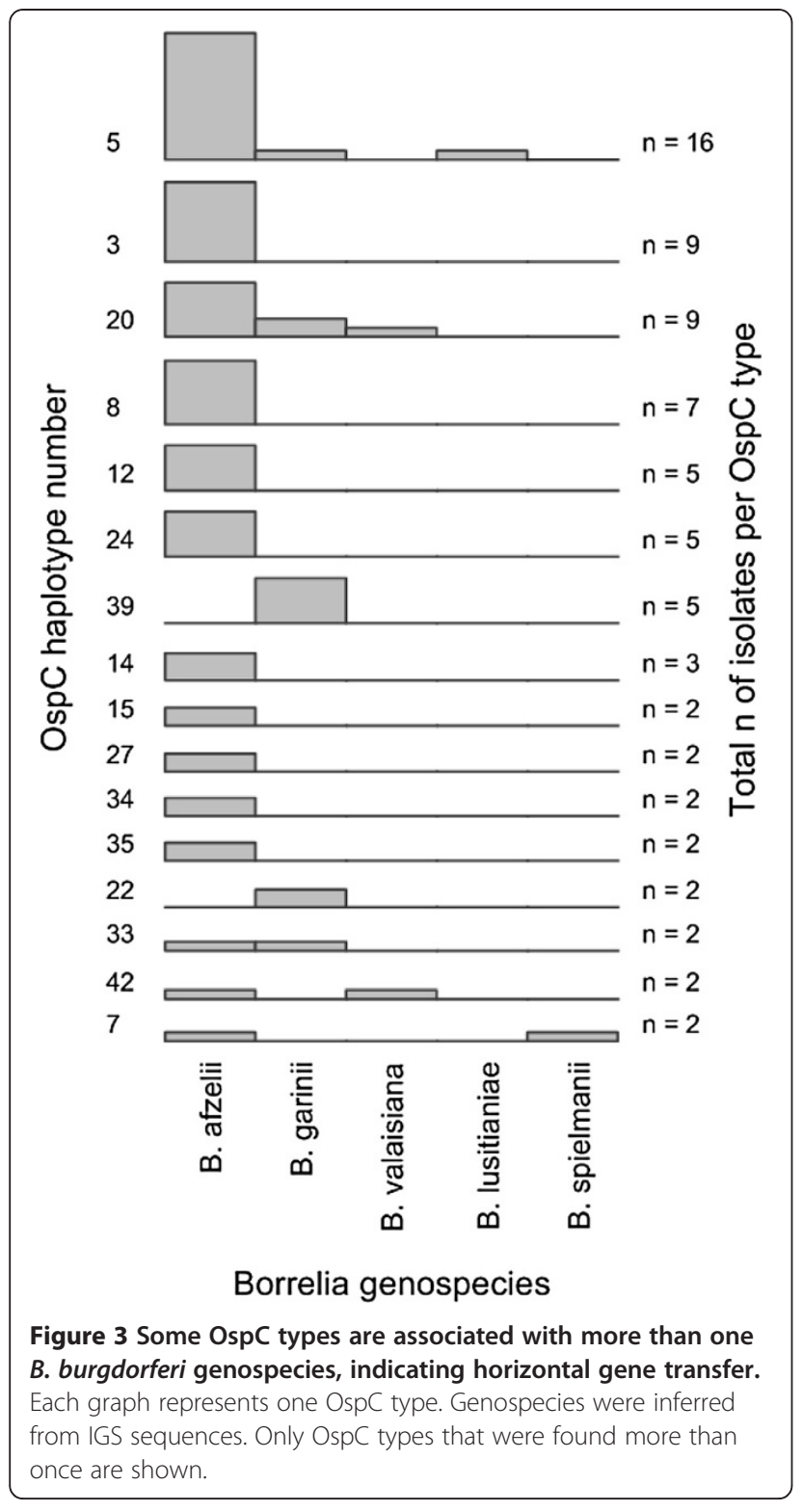

Rijeka study areas had B. burgdorferi sl sequences which were not found in any of the ticks can be explained by the lack of tick data from those study areas. These data highlight the importance of tick sampling representative for a certain study population. As Lyme borreliosis can be acquired by bites from adult as well as nymphal I. ricinus, a further potential bias lies in the testing of adult ticks only. Adult ticks might have obtained B. burgdorferi sl during feeding as nymphs. Hosts that fed nymphs and larvae might slightly differ and thus adult ticks might be infested with a different subpopulation of B. burgdorferi sl than nymphs.

It is unlikely that the propensity to cause EM or the invasiveness of Borrelia strains is determined by a single virulence factor alone. We rather hypothesize that a combination of different virulence factors encoded on the chromosome as well as on several plasmids of Borrelia determine the pathogenicity and invasiveness of different strains. Recently, a study showed that expression of $\operatorname{sp} p C$ is regulated by a gene on a different plasmid [38]. This emphasizes that the virulence of a certain strain, but also its fitness in a certain ecosystem, is a product of a complex system of genes interacting with each other and that it might be influenced by horizontal gene transfer. Even with the limited number of samples $(n=99)$ for which both genetic markers were available, five ospC types were found in more than one genospecies. This shows that substantial horizontal gene transfer takes place between different genospecies. Plasmid transfer has been reported before and shows that the apparent clonality seen in North American B. burgdorferi ss is lacking in European genospecies [10,39-41].

The current study shows that different Borrelia IGS and osp $C$ types exhibit different propensities to cause EM. We cannot exclude, however, that the genotypes involved in EM differ by location and in time. Furthermore, $B$. burgdorferi sl with IGS and ospC types identified to have a low propensity to cause EM here, could omit this early manifestation of Lyme borreliosis and directly cause disseminated manifestations of the disease. Besides the genetic constitution of $B$. burgdorferi sl, other explanations for the variability in the clinical manifestations of Lyme borreliosis are the (genetic) constitution of the human host [42,43], co-infections with other pathogens [44-46] and the bacterial load of ticks. However, the current study only focuses on genetic aspects of B. burgdorferi sl.

In future studies other sample types, such as cerebrospinal fluid or arthritis isolates, could be analysed in a similar way as was done here. The two markers used here are not directly responsible for EM formation and efforts should be undertaken to identify virulence factors that determine clinical manifestations. Once virulence genes are identified, risk assessment for Lyme borreliosis could be improved by including only human pathogenic B. burgdorferi sl into the risk assessment. In future, this information might help in the design of ecological interventions to reduce Lyme borreliosis risk, the improvement of serology-based diagnosis by use of more specific antigens or for the development of specialised vaccines targeting virulent $B$. burgdorferi sl strains.

\section{Conclusion}

We identified a strong correlation of certain IGS and $o s p C$ types with formation of erythema migrans. This indicates that only a small subset of B. burgdorferi sl carried by ticks in the wild actually cause EM. Other genotypes either may be apathogenic for humans or may cause Lyme borreliosis without the typical first sign of infection. 
However, as we restricted our investigation to an early form of Lyme borreliosis, it cannot be excluded that other $B$. burgdorferi sl genospecies and genotypes, not associated with EM, might omit this early sign of Lyme borreliosis and still lead to disseminated infections. This should be investigated using a broader range of patient materials from a broader geographic area.

\section{Additional files}

Additional file 1: Figure S1. Alignment of all identified IGS haplotypes with B. afzelii PKo (CP002933), REGION: 438843-439146.

Additional file 2: Figure S2. Alignment of all identified OspC haplotypes with B. afzelii PKo (CP002934), REGION: 17065-17463.

\section{Competing interests}

The authors declare that they have no competing interests.

\section{Authors' contributions}

NP and HS designed the study and NP collected samples. ETK and PH carried out laboratory experiments. ETK, KT and HS conducted genetic and statistic data analyses. ETK, HS and MK wrote the final manuscript. All authors read and approved the final version of the manuscript.

\section{Acknowledgements}

We would like to thank Jolyon Medlock for determining Haemaphysalis and Dermacentor ticks to species level.

This work was supported by the Dutch Food and Consumer Product Safety Authority and by the Ministry of Health, Welfare and Sport.

\section{Author details}

${ }^{1}$ Centre for Infectious Disease Control, National Institute for Public Health and the Environment (RIVM), P.O. Box 1, Bilthoven 3720BA, Netherlands. 'Department of Infectious Diseases, General Hospital "Dr. Josip Bencevic", Slavonski Brod, Croatia. 'Department of Virology, Erasmus Medical Centre, Rotterdam, Netherlands.

Received: 17 December 2012 Accepted: 9 January 2013

Published: 22 January 2013

\section{References}

1. Rudenko N, Golovchenko M, Grubhoffer L, Oliver JH Jr: Updates on Borrelia burgdorferi sensu lato complex with respect to public health. Ticks Tick Borne Dis 2011, 2:123-128.

2. Aucott JN, Seifter A, Rebman AW: Probable late lyme disease: a variant manifestation of untreated Borrelia burgdorferi infection. BMC Infect Dis 2012, 12:173.

3. Balmelli T, Piffaretti JC: Association between different clinical manifestations of Lyme disease and different species of Borrelia burgdorferi sensu lato. Res Microbiol 1995, 146:329-340.

4. van Dam AP, Kuiper H, Vos K, Widjojokusumo A, de Jongh BM, Spanjaard L, Ramselaar AC, Kramer MD, Dankert J: Different genospecies of Borrelia burgdorferi are associated with distinct clinical manifestations of Lyme borreliosis. Clin Infect Dis 1993, 17:708-717.

5. Ornstein K, Berglund J, Bergstrom S, Norrby R, Barbour AG: Three major Lyme Borrelia genospecies (Borrelia burgdorferi sensu stricto, B. afzelii and B. garinii) identified by PCR in cerebrospinal fluid from patients with neuroborreliosis in Sweden. Scand J Infect Dis 2002, 34:341-346.

6. Rijpkema SG, Tazelaar DJ, Molkenboer MJ, Noordhoek GT, Plantinga G, Schouls LM, Schellekens JF: Detection of Borrelia afzelii, Borrelia burgdorferi sensu stricto, Borrelia garinii and group VS116 by PCR in skin biopsies of patients with erythema migrans and acrodermatitis chronica atrophicans. Clin Microbiol Infect 1997, 3:109-116.

7. Picken RN, Strle F, Picken MM, Ruzic-Sabljic E, Maraspin V, Lotric-Furlan S, Cimperman J: Identification of three species of Borrelia burgdorferi sensu lato (B. burgdorferi sensu stricto, B. garinii, and B. afzelii) among isolates from acrodermatitis chronica atrophicans lesions. J Invest Dermatol 1998, 110:211-214

8. Seinost G, Dykhuizen DE, Dattwyler RJ, Golde WT, Dunn JJ, Wang IN, Wormser GP, Schriefer ME, Luft BJ: Four clones of Borrelia burgdorferi sensu stricto cause invasive infection in humans. Infect Immun 1999, 67:3518-3524

9. Baranton G, Seinost G, Theodore G, Postic D, Dykhuizen D: Distinct levels of genetic diversity of Borrelia burgdorferi are associated with different aspects of pathogenicity. Res Microbiol 2001, 152:149-156.

10. Wormser GP, Brisson D, Liveris D, Hanincova K, Sandigursky S, Nowakowski J, Nadelman RB, Ludin S, Schwartz I: Borrelia burgdorferi genotype predicts the capacity for hematogenous dissemination during early Lyme disease. J Infect Dis 2008, 198:1358-1364.

11. Eiffert H, Ohlenbusch A, Christen HJ, Thomssen R, Spielman A, Matuschka FR: Nondifferentiation between Lyme disease spirochetes from vector ticks and human cerebrospinal fluid. J Infect Dis 1995, 171:476-479.

12. Ryffel K, Peter O, Rutti B, Suard A, Dayer E: Scored antibody reactivity determined by immunoblotting shows an association between clinical manifestations and presence of Borrelia burgdorferi sensu stricto, B. garinii, B. afzelii, and B. valaisiana in humans. J Clin Microbiol 1999, 37:4086-4092.

13. Wang G, van Dam AP, Schwartz I, Dankert J: Molecular typing of Borrelia burgdorferi sensu lato: taxonomic, epidemiological, and clinical implications. Clin Microbiol Rev 1999, 12:633-653.

14. Schaarschmidt D, Oehme R, Kimmig P, Hesch RD, Englisch S: Detection and molecular typing of Borrelia burgdorferi sensu lato in Ixodes ricinus ticks and in different patient samples from southwest Germany. Eur J Epidemiol 2001, 17:1067-1074.

15. Masuzawa T, Komikado T, Iwaki A, Suzuki H, Kaneda K, Yanagihara Y: Characterization of Borrelia sp. isolated from Ixodes tanuki, I. turdus, and I. columnae in Japan by restriction fragment length polymorphism of rrf (5S)rrl (23S) intergenic spacer amplicons. FEMS Microbiol Lett 1996, 142:77-83.

16. Tijsse-Klasen E, Fonville M, Reimerink JH, Spitzen-van der Sluijs A, Sprong H: Role of sand lizards in the ecology of Lyme and other tick-borne diseases in the Netherlands. Parasit Vectors 2010, 3:42.

17. Jenkins A, Hvidsten D, Matussek A, Lindgren PE, Stuen S, Kristiansen BE: Borrelia burgdorferi sensu lato in Ixodes ricinus ticks from Norway: evaluation of a PCR test targeting the chromosomal flaB gene. Exp Appl Acarol 2012, 58:431-439.

18. Margos G, Vollmer SA, Ogden NH, Fish D: Population genetics, taxonomy, phylogeny and evolution of Borrelia burgdorferi sensu lato. Infect Genet Evol 2011, 11:1545-1563.

19. Postic D, Assous MV, Grimont PA, Baranton G: Diversity of Borrelia burgdorferi sensu lato evidenced by restriction fragment length polymorphism of rrf (5S)-rrl (23S) intergenic spacer amplicons. Int J Syst Bacteriol 1994, 44:743-752

20. Tijsse-Klasen E, Jacobs JJ, Swart A, Fonville M, Reimerink JH, Brandenburg $\mathrm{AH}$, van der Giessen JW, Hofhuis A, Sprong H: Small risk of developing symptomatic tick-borne diseases following a tick bite in the Netherlands. Parasit Vectors 2011, 4:17.

21. Kenedy MR, Lenhart TR, Akins DR: The role of Borrelia burgdorferi outer surface proteins. FEMS Immunol Med Microbiol 2012, 66:1-19.

22. Grimm D, Tilly K, Byram R, Stewart PE, Krum JG, Bueschel DM, Schwan TG, Policastro PF, Elias AF, Rosa PA: Outer-surface protein C of the Lyme disease spirochete: a protein induced in ticks for infection of mammals. Proc Natl Acad Sci U S A 2004, 101:3142-3147.

23. Tilly K, Casjens S, Stevenson B, Bono JL, Samuels DS, Hogan D, Rosa P: The Borrelia burgdorferi circular plasmid cp26: conservation of plasmid structure and targeted inactivation of the ospC gene. Mol Microbiol 1997, 25:361-373.

24. Tilly K, Krum JG, Bestor A, Jewett MW, Grimm D, Bueschel D, Byram R, Dorward D, Vanraden MJ, Stewart P, Rosa P: Borrelia burgdorferi OspC protein required exclusively in a crucial early stage of mammalian infection. Infect Immun 2006, 74:3554-3564.

25. Alghaferi MY, Anderson JM, Park J, Auwaerter PG, Aucott JN, Norris DE, Dumler JS: Borrelia burgdorferi ospC heterogeneity among human and murine isolates from a defined region of northern Maryland and southern Pennsylvania: lack of correlation with invasive and noninvasive genotypes. J Clin Microbiol 2005, 43:1879-1884.

26. Earnhart CG, Buckles EL, Dumler JS, Marconi RT: Demonstration of OspC type diversity in invasive human lyme disease isolates and identification of previously uncharacterized epitopes that define the specificity of the OspC murine antibody response. Infect Immun 2005, 73:7869-7877. 
27. Wielinga PR, Gaasenbeek C, Fonville M, de Boer A, de Vries A, Dimmers W, Akkerhuis Op Jagers G, Schouls LM, Borgsteede F, van der Giessen JW: Longitudinal analysis of tick densities and Borrelia, Anaplasma, and Ehrlichia infections of Ixodes ricinus ticks in different habitat areas in The Netherlands. Appl Environ Microbiol 2006, 72:7594-7601.

28. Heylen D, Tijsse E, Fonville M, Matthysen E, Sprong H: Transmission dynamics of Borrelia burgdorferi s.l. in a bird tick community. Environ Microbiol 2012, In press.

29. Wang IN, Dykhuizen DE, Qiu W, Dunn JJ, Bosler EM, Luft BJ: Genetic diversity of ospC in a local population of Borrelia burgdorferi sensu stricto. Genetics 1999, 151:15-30.

30. Tomanovic S, Chochlakis D, Radulovic Z, Milutinovic M, Cakic S, Mihaljica D Tselentis Y, Psaroulaki A: Analysis of pathogen co-occurrence in hostseeking adult hard ticks from Serbia. Exp Appl Acarol 2012, In press.

31. Rijpkema S, Golubic D, Molkenboer M, Verbeek-De Kruif N, Schellekens J: Identification of four genomic groups of Borrelia burgdorferi sensu lato in Ixodes ricinus ticks collected in a Lyme borreliosis endemic region of northern Croatia. Exp Appl Acarol 1996, 20:23-30.

32. Milutinovic M, Radulovic Ž, Tomanovic S, Tomanovic Z: Seasonal distribution of Borreliae in Ixodes ricinus ticks in the Belgrade Region, Serbia. Archives of Biological Science, Belgrade 2006, 58:183-186.

33. Schwarz A, Honig V, Vavruskova Z, Grubhoffer L, Balczun C, Albring A, Schaub $\mathrm{GA}$ : Abundance of Ixodes ricinus and prevalence of Borrelia burgdorferi s.l. in the nature reserve Siebengebirge, Germany, in comparison to three former studies from 1978 onwards. Parasit Vectors 2012, 5:268.

34. Egyed L, Elo P, Sreter-Lancz Z, Szell Z, Balogh Z, Sreter T: Seasonal activity and tick-borne pathogen infection rates of Ixodes ricinus ticks in Hungary. Ticks Tick Borne Dis 2012, 3:90-94.

35. Rigo K, Gyuranecz M, Toth AG, Foldvari G: Detection of Borrelia burgdorferi sensu Lato and Anaplasma phagocytophilum in small mammals and ectoparasites in Hungary. Vector Borne Zoonotic Dis 2011, 11:1499-1501.

36. Dykhuizen DE, Brisson D, Sandigursky S, Wormser GP, Nowakowski J, Nadelman RB, Schwartz I: The propensity of different Borrelia burgdorferi sensu stricto genotypes to cause disseminated infections in humans. AmJTrop Med Hyg 2008, 78:806-810.

37. Lagal V, Postic D, Ruzic-Sabljic E, Baranton G: Genetic diversity among Borrelia strains determined by single-strand conformation polymorphism analysis of the ospC gene and its association with invasiveness. J Clin Microbiol 2003, 41:5059-5065.

38. Sarkar A, Hayes BM, Dulebohn DP, Rosa PA: Regulation of the virulence determinant OspC by bbd18 on linear plasmid Ip17 of Borrelia burgdorferi. J Bacteriol 2011, 193:5365-5373.

39. Jones KL, McHugh GA, Glickstein LJ, Steere AC: Analysis of Borrelia burgdorferi genotypes in patients with Lyme arthritis: High frequency of ribosomal RNA intergenic spacer type 1 strains in antibiotic-refractory arthritis. Arthritis Rheum 2009, 60:2174-2182.

40. Travinsky B, Bunikis J, Barbour AG: Geographic differences in genetic locus linkages for Borrelia burgdorferi. Emerg Infect Dis 2010, 16:1147-1150.

41. Jauris-Heipke S, Liegl G, Preac-Mursic V, Rossler D, Schwab E, Soutschek E, Will G, Wilske B: Molecular analysis of genes encoding outer surface protein C (OspC) of Borrelia burgdorferi sensu lato: relationship to ospA genotype and evidence of lateral gene exchange of ospC. J Clin Microbiol 1995, 33:1860-1866.

42. Dickman S: Possible cause found for Lyme arthritis. Science 1998 281:631-632.

43. Hemmer B, Gran B, Zhao Y, Marques A, Pascal J, Tzou A, Kondo T, Cortese I, Bielekova B, Straus SE, et al: Identification of candidate T-cell epitopes and molecular mimics in chronic Lyme disease. Nat Med 1999, 5:1375-1382.

44. Eskow E, Adelson ME, Rao RV, Mordechai E: Evidence for disseminated Mycoplasma fermentans in New Jersey residents with antecedent tick attachment and subsequent musculoskeletal symptoms. J Clin Rheumatol 2003, 9:77-87.

45. Flicek BF: Rickettsial and other tick-borne infections. Crit Care Nurs Clin North Am 2007, 19:27-38.

46. Swanson SJ, Neitzel D, Reed KD, Belongia EA: Coinfections acquired from ixodes ticks. Clin Microbiol Rev 2006, 19:708-727.

doi:10.1186/1756-3305-6-23

Cite this article as: Tijsse-Klasen et al:: Ability to cause erythema migrans differs between Borrelia burgdorferi sensu lato isolates. Parasites \& Vectors 2013 6:23.

\section{Submit your next manuscript to BioMed Central and take full advantage of:}

- Convenient online submission

- Thorough peer review

- No space constraints or color figure charges

- Immediate publication on acceptance

- Inclusion in PubMed, CAS, Scopus and Google Scholar

- Research which is freely available for redistribution 